\title{
El efecto anti-hipertensivo de Angiotensina-(1-9) es mediado por aumento temprano de la diuresis y natriuresis
}

\author{
Jackeline Moya ${ }^{1}$, Ulises Novoa ${ }^{2}$, Nicolás Escudero ${ }^{1}$, Víctor Barrientos ${ }^{3}$, Mario Chiong $^{4}$, Sergio Lavandero ${ }^{4,5}$, \\ Luis Michea ${ }^{3}$, Jorge Jalil', María Paz Ocaranza ${ }^{1,6}$.

\begin{abstract}
1 División de Enfermedades. Cardiovasculares, Escuela de Medicina, Pontificia Universidad Católica de Chile. 2 Departamento de Cs. Básicas Biomédicas, Universidad de Talca

4 Advanced Center for Chronic Diseases (ACCDiS), Centro de Estudios Moleculares de la Célula, Facultad de Ciencias Quimicas y Farmaceuticas \& Facultad de Medicina, Universidad de Chile

5 Department of Internal Medicine (Cardiology Division), University of Texas Southwestern Medical Center, Dallas, Texas, USA
\end{abstract} \\ 3 Laboratorio de Fisiologia Integrativa, Faculta de Medicina, Universidad de Chile \\ 6 Advanced Center for Chronic Diseases (ACCDiS), Facultad de Medicina, Pontificia Universidad Católica de Chile.
}

Introducción: Angiotensina (Ang)-(1-9) posee propiedades anti-hipertensivas y efecto protector a nivel cardiovascular en ratas hipertensas. Sin embargo, se desconoce si estos efectos están asociados a un mecanismo de desbalance de sodio a nivel renal.

Objetivo: Determinar si el efecto anti-hipertensivo de Ang-(1-9) está asociado a un mecanismo diurético-natriurético.

Método: Ratas macho Sprague Dawley $(200 \pm 10 \mathrm{~g})$ fueron aleatorizadas para recibir Ang II (400 ng/kgmin) vía bomba osmótica. Como control se utilizaron ratas con operación sham $(\mathrm{n}=18)$. Después de 2 semanas desde la instalación de bomba, las ratas Sham e hipertensas fueron randomizadas para recibir vehículo ( $\mathrm{n}=10)$, Ang(1-9) $(602 \mathrm{ng} / \mathrm{kg} / \mathrm{min}, \mathrm{n}=17)$ o una co-administración de Ang-(1-9) y A779 (100 ng kg-1min-1, n=7 bloqueador del receptor MAS) por 2 semanas.

\section{Resultados:}

Se determinó la presión arterial sistólica (PAS), masa ventricular relativa (MVR), área y perímetro de los cardiomiocitos (AC y PC) y la fracción volumétrica de colágeno total (FVCT). Para evaluar la diuresis y natriuresis se utilizaron ratas normotensas que fueron randomizadas para recibir vehículo ( $\mathrm{n}=8$ ) o Ang-(1-9) (600 ng·Kg-1·min-1, n=8) por 6 días. Se observó un incremento significativo $(\mathrm{p}<0.05)$ de PAS $(33 \%)$, MVR (17\%), AC (64\%), PC (20\%), FVCT (46\%). La administración crónica de Ang-(1-9) disminuyó PAS (20\%), MVR (13\%), AC (35\%), PC (20\%) y FVCT (20\%). Estos efectos no fueron mediados por el receptor MAS. Al comparar las ratas normotensas tratadas con vehículo o Ang-(1-9), se observó un aumento significativo de la diuresis y natriuresis en los días 2 y 3 en los animales con infusión de Ang-(1-9).

Conclusión: Ang-(1-9) reduce la hipertensión y el remodelamiento cardíaco en ratas hipertensas. En animales normotensos se demostró que el tratamiento con Ang-(1-9)-induce diuresis y natriuresis. Este es el primer reporte que señala que el efecto de Ang-(1-9) está asociado a una regulación del sodio a nivel renal.

Financiamiento: Fondef D1111122, Fondap 15130011.

\section{Correspondencia:}

María Paz Ocaranza, División de Enfermedades

Cardiovasculares. Facultad de Medicina. Pontificia Universidad

Católica de Chile. Tel: +562-3543407, Fax: +562-6321924.

mail: mocaran@med.puc.cl 


\section{Cardiac Arrest associated to Intramural Hematoma of the Anterior Descending Coronary Artery}

Angiotensin-(1-9) has anti-hypertensive properties and protective cardiovascular effect in hypertensive rats. However, it is unknown whether its effects are related to a kidney mechanism to balance sodium.

Aim: To determine if the anti-hypertensive effect of Ang-(1-9) is associated to a diuretic-natriuretic mechanism.

Method: Sprague Dawley male rats $(200 \pm 10$ grs $)$ were randomized to receive Angiotensin II by osmotic pump (400 $\mathrm{ng} / \mathrm{kg} / \mathrm{min})$. Sham operated rats were utilized as control $(n=18)$. Two weeks after pump setting, Sham rats with hypertension were randomized to receive placebo $(\mathrm{n}=10)$, Ang-(1-9)(602 ng/kg/min, $\mathrm{n}=17$ ) or Ang-(1-9) plus A779 (Ang-(1-7) Receptor Mas blocker, 100ng/kg-1min-1, n=7) co-administration for two weeks. Arterial systolic pressure (PAS), ventricular relative mass (MVR), cardiomyocytes area and perimeter (AC and $\mathrm{PC}$ ) and total collagen volume fraction (FVCT) were measured. Normotensive rats were utilized to evaluate diuresis and natriuresis which were randomized to receive placebo $(n=8)$ or Ang-(19) $(600 \mathrm{ng} / \mathrm{kg}-1 / \mathrm{min}-1, \mathrm{n}=8)$ for six days.

Results: It was observed a significant rise $(\mathrm{p}<0.05)$ of PAS (33\%), MVR (17\%), AC (64\%), PC (26\%), FVCT $(46 \%)$ was observed. Chronic administration of Ang-(1-9) reduced PAS (20\%), MVR (13\%), AC (35\%), PC (20\%) and FCVT (20\%). All those effects were not mediated by Mas receptor. A significant raise was observed of diuresis and natriuresis at the second and third day of treatment in rats receiving Ang-(19) in comparison with normotensive rats treated with placebo.

Conclusion: Ang-(1-9) reduces hypertension and cardiac remodeling in hypertensive rats. Ang-(1-9) induces natriuresis and diuresis in normotensive rats. This is the first report showing that Ang-(1-9) is associated to sodium balance in the kidney.

Keywords: Hypertension arterial; Renin-Angiotensin System; Angiotensin (1-9); Diuresis; Natriuresis. 


\section{Introducción:}

El desbalance del Sistema Renina Angiotensina (SRA) es uno de los principales factores fisiopatológicos asociados al desarrollo de la hipertensión (HTA ${ }^{1}$ Específicamente, la sobreactivación de la vía clásica del SRA conformada por la enzima convertidora de angiotensina I (ECA) y su producto angiotensina (Ang) II. La Ang II al unirse a su receptor AT1 (RAT1) activa las vías de señalización que desencadenan un aumento de la presión arterial (PA) y cambios celulares, no celulares y funcionales en los distintos tejidos blanco como corazón y vasos, entre otros². El descubrimiento de la ECA homóloga (ECA2) ${ }^{3-4}$ estableció una vía paralela del SRA. En esta vía, ECA2 compite con ECA por la hidrólisis de Ang I para formar Ang(1-9) ${ }^{4}$, además ECA2 produce Ang-(1-7), a partir de Ang II $^{5-6}$. Hasta la fecha, la mayor parte de la evidencia se ha basado en Ang-(1-7), péptido con propiedades antagónicas a los efectos vasopresor, proliferativo, profibrótico, y protrombótico de Ang II $^{7}$. Estudios experimentales y clínicos han demostrado el papel del eje Ang- (1-7)/ECA2/MAS en el balance de la presión arterial y en la regulación de la función $\mathrm{CV}$ y renal ${ }^{8}$. Evidencias adicionales sugieren que la disminución en la expresión y actividad del eje clásico vasopressor del SRA puede ser un factor crítico en mediar la progresión de la enfermedad CV y renal. Por lo tanto, estos antecedentes proponen un rol contrarregulador del eje Ang-(1-7)/ECA2/MAShacia el eje ECA/Ang I/receptor AT1 en el sistema Renina-Angiotensina-Aldosterona (SRA) $)^{7-8}$.

Respecto a Ang-1-9, en estudios previos realizados por nuestro grupo hemos propuesto que el péptido Ang-(19) podría actuar como un contrarregulador de Ang II más efectivo que Ang-(1-7) ${ }^{9-10}$. Además, hemos determinado que Ang-(1-9) previene el desarrollo de hipertrofia cardíaca in vitro e in vivo, independiente del receptor de Ang-(17), MAS $^{10}$. En otro estudio hemos observado una disminución significativa de los niveles de Ang-(1-9) y de ECA2 en la pared aórtica en el modelo de animales con HTA y remodelamiento cardiovascular hipertensivo ${ }^{11-12}$.

Nuestros primeros resultados del efecto de Ang-(1-9) sobre la HTA se observó en el modelo experimental de ratas con HTA mediante infusión de Ang II ${ }^{13}$ y en el modelo de ratas con niveles géticamente determinados de Ang $\mathrm{II}^{14}$. En tales estudios, la administración crónica de Ang-(1-9) previno y disminuyó la HTA y el remodelamiento cardiovascular secundario. Tales efectos de Ang-(1-9) fueron directos y no mediados por Ang-(1-7) ${ }^{13-14}$. Estos hallazgos, sumado a que Ang-(1-9) es parte de un eje opuesto al SRA clásico, apoyan el concepto de que Ang-(1-9) podría ser un importante blanco terapéutico para el tratamiento de la HTA. Por el momento, se desconoce si su efecto anti-hipertensivo está asociado a un mecanismo diurético-natriurético. Por lo tanto, el objetivo de este estudio fue determinar si el efecto anti-hipertensivo de Ang-(1-9) está asociado a un mecanismo diurético-natriurético.

\section{Materiales y métodos}

Esta investigación se realizó según la "Guía para el cuidado y uso de animales de laboratorio" publicado por el "National Health Institute" (NIH No 85-23, 1985) y aprobada por la Comisión de Investigación de la Facultad de Medicina de la Pontificia Universidad Católica de Chile.

1. Modelo experimental de hipertensión: Ratas macho Sprague Dawley de 200 \pm 10 g se randomizaron a recibir suero fisiológico (Sham, control normotenso) o Ang II (400 ng/kg min) por bomba osmótica vía yugular ${ }^{15}$. Después de 2 semanas desde la primera bomba Alzet, las ratas Sham o Ang II fueron randomizadas para recibir en el caso de las Sham, Ang-(1-9) (602 ng/Kg min). MientSRA que las ratas hipertensas con PAS $\geq 140 \mathrm{mmHg}$ se randomizaron para recibir Ang-(1-9) (602 ng/Kg min) o una coadministración de Ang-(1-9) y A779 (100 ng/Kg min y antagonista del receptor MASde Ang-(1-7)) a un flujo de $0.5 \mathrm{~L} /$ hr por 2 semanas mediante una minibomba Alzet.

\section{Modelo experimental para medir diuresis/natriure-}

sis: Para determinar si el mecanismo de Ang-(1-9) podría implicar una acción diurética se evaluó la diuresis y natriuresis en ratas controles. Para esto se usaron ratas macho Sprague Dawley normotensas de $200 \pm 10 \mathrm{~g}$, las que fueron alimentadas con una dieta estándar que contenía $\mathrm{NaCl}$ 2,3 $\mathrm{g} / \mathrm{kg}$. Tres días antes de iniciar el protocolo experimental, las ratas se colocaron en jaulas metabólicas por 48 hoSRA, para adaptación. Para determinar la diuresis y excreción basal de sodio urinario se colectó la orina de 24 hoSRA. Posteriormente, las ratas fueron randomizadas a recibir vehículo o Ang-(1-9) [600 ng·Kg-1·min-1] por 6 días infundidos por bomba osmótica Alzet conectada a la yugular del animal. La orina diaria se colectó por 5 días, empezando 24 hoSRA después de la implantación de la mini bomba osmótica.

3. Parámetros hemodinámicos: La PAS fué medida semanalmente a través de un método indirecto no invasivo CODA 2 tail-cuff ${ }^{16}$. También se evaluó semanalmente la masa corporal (MC) de los animales. Después que los animales fueron eutanasiados, se midió la masa ventricular 
Figura 1. Efecto de la administración crónica de Ang-(1-9) en la presión arterial sistólica de ratas con HTA inducida con Ang II. Abreviaturas: PAS = Presión Arterial Sistólica, Sham = ratas normotensas, Sham Ang 1-9= ratas normotensas tratadas con Ang-(1-9), Ang II = modelo hipertenso por administración de Angiotensina II, Ang II/1-9 = ratas hipertensas tratadas con Ang(1-9), Angll/1-9/A779 = ratas hipertensas tratadas con Ang-(1-9) + A779. Los cambios de PAS en cada grupo fueron determinados por un método tail-cuff. Grafico de PAS en el transcurso de las 4 semanas de tratamiento. Los resultados fueron graficados según el promedio \pm SEM. $N=7-11$. * $p \leq 0,05$ Sham y \# $p \leq 0,05$ Ang II; después de ANOVA para comparación entre grupos.

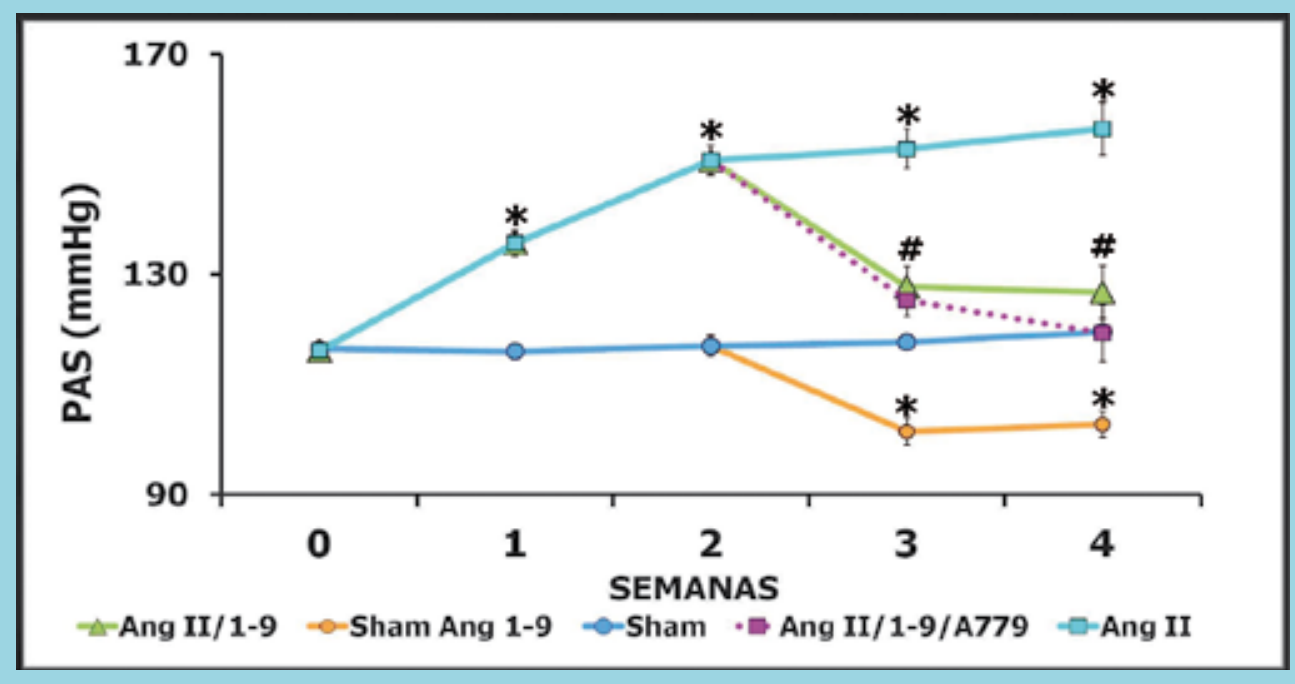

(MV) y el largo de la tibia (LT), para determinar la masa ventricular relativa (MVR)

4. Obtención de tejidos: Los animales se anestesiaron con Ketamina ( $35 \mathrm{mg} / \mathrm{Kg}$ peso) y Xilacina ( $7 \mathrm{mg} / \mathrm{Kg}$ peso), vía intra peritonal. El corazón se extrajo rápidamente y se lavó exhaustivamente en suero fisiológico, se congeló en nitrógeno líquido y se guardó a -800C hasta su procesamiento. Además, parte del miocardio fue fijada en Bouin, desde donde se obtuvieron cortes de tejido ventricular ${ }^{17}$.

\section{Análisis morfométrico de hipertrofia cardíaca:} Cortes tSRAversales de $5 \mu \mathrm{m}$ de grosor, se tiñeron con Hematoxilina-Eosina. Las imágenes fueron tomadas mediante una cámara acoplada a un microscopio (Nikon eclipse E400) y cuantificadas utilizando un software (Nis-Element). Se midió el área de los cardiomiocitos (AC, $\mu \mathrm{m} 2)$ y perímetro de los cardiomiocitos $(\mathrm{PC}, \mu \mathrm{m})^{18}$. Se analizaron al menos 70 imágenes celulares por animal, las que se seleccionaron aleatoriamente. Además, el grado de hipertrofia cardíaca se evaluó de acuerdo a los parámetros de masa corporal (MC, gr) y masa ventricular (MV, gr).

6. Análisis morfométrico de fibrosis cardíaca: Cortes transversales de VI de $5 \mu \mathrm{m}$ de grosor fueron teñidos con rojo picrosirio ${ }^{19-20}$. Las imágenes fueron captadas con una cámara digital acoplada a un microscopio. Con el aumento de $20 \mathrm{X}$ se tomaron fotos sucesivas hasta completar entre 60-80 fotos, aproximadamente. Finalmente, se fijó el lente en una zona carente de tejido y se captó una foto blanco, con el propósito de identificar los espacios sin tejido. Estas imágenes fueron analizadas con el programa Matlab diseñado para la medición de Colágeno, bajo protocolo establecido en el Laboratorio ${ }^{20}$. Finalmente, se determinó el promedio de la fracción volumétrica de colágeno total (FVCT, \%).

7. Determinación de la concentración de sodio urinario. Se determinó por un electrodo ion-selectivo (KoneLab20, Thermo Scientific).

8. Expresión de resultados y análisis estadístico: Los datos obtenidos se expresaron como promedio \pm error estándar de la media (SEM). Cada grupo experimental estuvo constituido por $\mathrm{N}$ entre 7-12 animales. Las comparaciones entre los grupos se realizaron mediante ANOVA de una vía, y test Newman keuls post hoc.

\section{Resultados}

1. Efecto de Ang-(1-9) en la HTA inducida por Ang II: En los animales hipertensos se observó un aumento significativo de la PAS a partir de la primera semana (sem) post implantación de bomba Alzet en comparación al grupo Sham (Figura 1). La administración crónica de Ang-(1- 


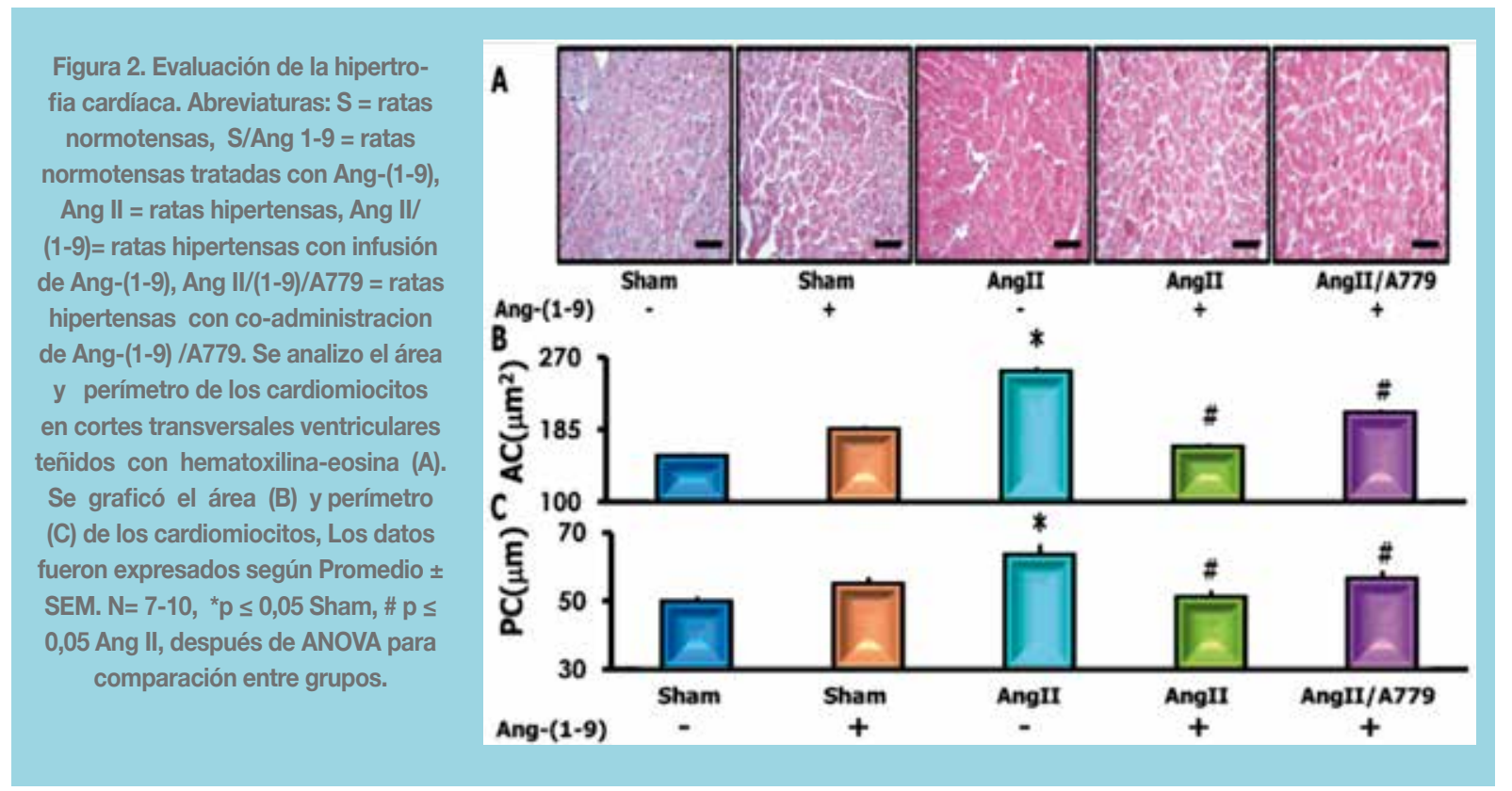

9) disminuyó significativamente la PAS en un $17 \%$ a la 1era sem post administración en comparación con los animales hipertensos. Estas disminuciones alcanzaron un $22 \%$ en la segunda sem post administración (Figura 1). La co-administración del bloqueo del receptor MAScon A779 también disminuyó la PAS en un $23 \%$ en comparación con los animales hipertensos por infusión de Ang II (Figura 1). La administración de Ang-(1-9) a los animales Sham produjo un efecto hipotensor disminuyendo en un $10 \%$ la PAS en comparación con su control normotenso.

2. Efecto del tratamiento en la Masa Corporal, Masa ventricular y Masa ventricular relativa de los animales: La masa corporal (MC) no mostró diferencias significativas entre los distintos grupos experimentales. MientSRA que en la MC final se apreció una disminución significativa en los modelos coinfundidos con Ang II y Ang-(19) y Ang II, Ang-(1-9) y A779 vssu control normotenso e hipertenso $(259 \pm 5$ y $267 \pm 17$ vs318 \pm 8 y $305 \pm 12)$. En comparación, las ratas normotensas tratadas con Ang(1-9) no mostraron diferencias en la MC final respecto a ratas controles e hipertensas (Tabla 1). La masa ventricular (MV) y la MVR mostró un aumento significativo en las ratas hipertensas con respecto a las ratas sham (MV: 1.15 \pm 0.01 vs0.99 \pm 0.01 , MVR: $30.4 \pm 0.4$ vs $25.9 \pm 0.4) . \mathrm{El}$ tratamiento con Ang-(1-9) y con A779 mostró un disminución significativa de la MV y MVR en comparación con los animales hipertensos (MV: $1.15 \pm 0.01$ vs $0.99 \pm 0.02$ o 0.95 \pm 0.05 , MVR: $30.4 \pm 0.4$ vs $26.5 \pm 0.7$ o $26.9 \pm 1.1)$. No se observaron diferencias entre los animales Sham/Ang-(1-9) y su control normotenso. El largo de la tibia no mostró diferencias entre los modelos experimentales (Tabla 1).

3. Efecto de Ang-(1-9) en la hipertrofia cardíaca: Los cortes de ventrículos (Figura 2A), mostraron un aumento significativo en el AC y PC en las ratas hipertensas con respecto a las ratas normotensas (AC: $253 \pm 3$ vs $154 \pm 1$ $\mu \mathrm{m} 2$; PC: $63 \pm 3$ vs $50 \pm 1 \mu \mathrm{m}$, respectivamente, (Figura 2 B y C)). Sin embargo, en las ratas hipertensas tratadas con Ang-(1-9) se observó una disminución significativa del AC y PC en comparación con las ratas hipertensas (AC: $165 \pm 2$ vs $253 \pm 3 \mu \mathrm{m} 2 ;$ PC: $51 \pm 2$ vs $63 \pm 3$ $\mu \mathrm{m}$, respectivamente, (Figura $2 \mathrm{~B}$ y C)). La coadministración de A779 no modificó el efecto antihipertrófico de Ang-(1-9) [AC: $205 \pm 2$ vs $253 \pm 3 \mu \mathrm{m} 2$; PC: $57 \pm 2$ vs $63 \pm 3 \mu \mathrm{m}$, respectivamente, (Figura $2 \mathrm{~B}$ y C)]. Los animales normotensos que recibieron Ang-(1-9), mostraron un aumento del AC y PC en comparación a su control Sham (AC: $186 \pm 1$ vs $154 \pm 1 \mu \mathrm{m} 2$; PC: $55 \pm 1$ vs $50 \pm 1 \mu \mathrm{m}$, respectivamente, (Figura $2 \mathrm{~B}$ y $\mathrm{C})$ ).

4. Ang-(1-9) en la fibrosis cardíaca: En relación a la fibrosis cardíaca, los cortes de ventrículos (Figura 3A), mostraron un aumento significativo en el FVCT en los animales hipertensos en comparación con los animales normotensos $(3.5 \pm 0.1$ vs $2.4 \pm 0.2$ (Figura $3 \mathrm{~B}))$. Al tratar las ratas hipertensas con Ang-(1-9), se observó una reversión de la FVCT $(2.8 \pm 0.2$ vs $3.5 \pm 0.1$ (Figura $3 \mathrm{~B})$ ) 


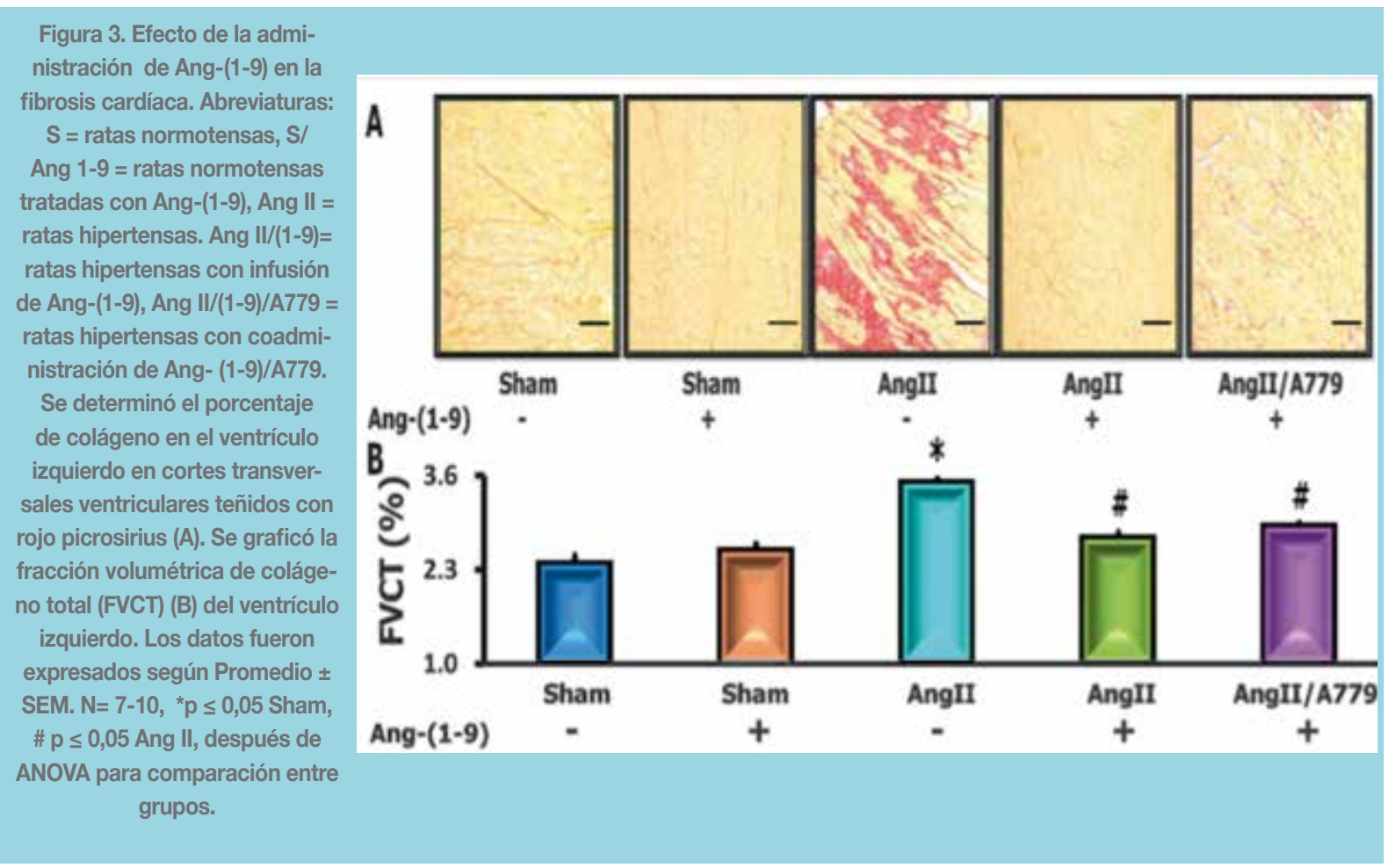

con respecto a las ratas hipertensas sin tratamiento. Los animales hipertensos que recibieron Ang-(1-9)/ A779 igualmente mostraron una disminución de la FVCT vs las ratas hipertensas tratados con vehículo $(2.9 \pm 0.2$ vs $3.5 \pm 0.1$. Figura 3B). No se observaron diferencias en la FVCT en las ratas normotensas tratadas con vehículo y con Ang-(1-9) [2.4 \pm 0.2 vs2.6 \pm 0.1 Figura 3B].

5. Ang-(1-9) en la Diuresis y Natriuresis: Las ratas normotensas tratadas con Ang-(1-9) mostraron un aumento significativo de la diuresis (Figura 4A) en los días 2, 3, y 6. Además se observó un aumento significativo de la natriuresis en el día 2 y 3 de la infusión con Ang-(1-9) (Figura 4B).

\section{Discusión}

Los principales resultados de este estudio fueron: 1) La administración crónica de Ang-(1-9) ejerció un importante efecto de disminuir la PA tanto en ratas normotensas como hipertensas, 2) En ratas hipertensas, Ang-(1-9) revirtió el remodelamiento cardíaco, evaluado por hipertrofia y fibrosis del ventrículo izquierdo. 3) Los efectos de Ang-(1-9) serían directos y no por su transformación a Ang-(1-7), 4) En ratas normotensas sanas, la infusión aguda con Ang-(1-9) indujo diuresis y natriuresis.

Los primeros antecedentes respecto al rol de la Ang-(1-9) en la HTA fueron aportados por Ocaranza et al (2011) ${ }^{11}$. Al respecto, se observó en ratas con HTA por sobrecarga de volumen (modelo DOCA-sal) y en ratas normotensas controles, que la inhibición de RhoA/Rho-kinasa (mecanismo de señalización intracelular que participa en el remodelamiento $\mathrm{CV}$ y renal patológico como también en la regulación de la PA) por fasudil disminuyó la PA y también aumentó la actividad de ECA2 en plasma y en la pared aórtica ${ }^{11}$. Junto con esto, fasudil disminuyó los niveles plasmáticos de Ang II y aumentó los niveles plasmáticos de Ang-(1-9), sin cambios en los niveles de Ang-(1-7 $)^{11}$. Por lo tanto, este novedoso efecto en el cual la inhibición de RhoA/Rho-kinasa aumenta la expresión de ECA2 y los niveles de Ang-(1-9), podría contribuir al efecto antihipertensivo de los inhibidores de RhoA/Rho-kinasa. Estos resultados sugirieron que en este modelo experimental, la HTA es más dependiente de la actividad de ECA2 y niveles de Ang-(1-9) que de la vía ECA/Ang II ${ }^{11}$.

Nuestro grupo de trabajo ya ha presentado antecedentes sobre el efecto antihipertensivo de Ang-(1-9) en modelos experimentales de prevención de la HTA inducida por infusion de Ang II $^{13}$ y en el modelo de ratas Goldblatt con niveles genéticamente determinados de ECA y Ang II $^{21}$. Nuestros resultados nuevamente evidenciaron la eficacia Ang-(1-9) en disminuir significativamente la HTA en ratas con HTA previa y sostenida por infusión de Ang II. Por 
Figura 4. Efecto de Ang-(1-9) en diuresis y natriuresis de ratas normotensas. La orina de las ratas normotensas fue recolectada en jaulas metabólicas inicialmente (día 0 , representa dos días previos a la implantación de la bomba osmótica) y dos días después de la administración de Ang-(1-9) se recolectó la orina periódicamente por 5 días. Todos los animales recibieron una dieta estándar. Se midió los niveles de Diuresis (A) y Natriuresis (B). Los datos fueron expresados según Promedio $\pm S E M$. $N=8$, * $p \leq 0,05$ Sham.
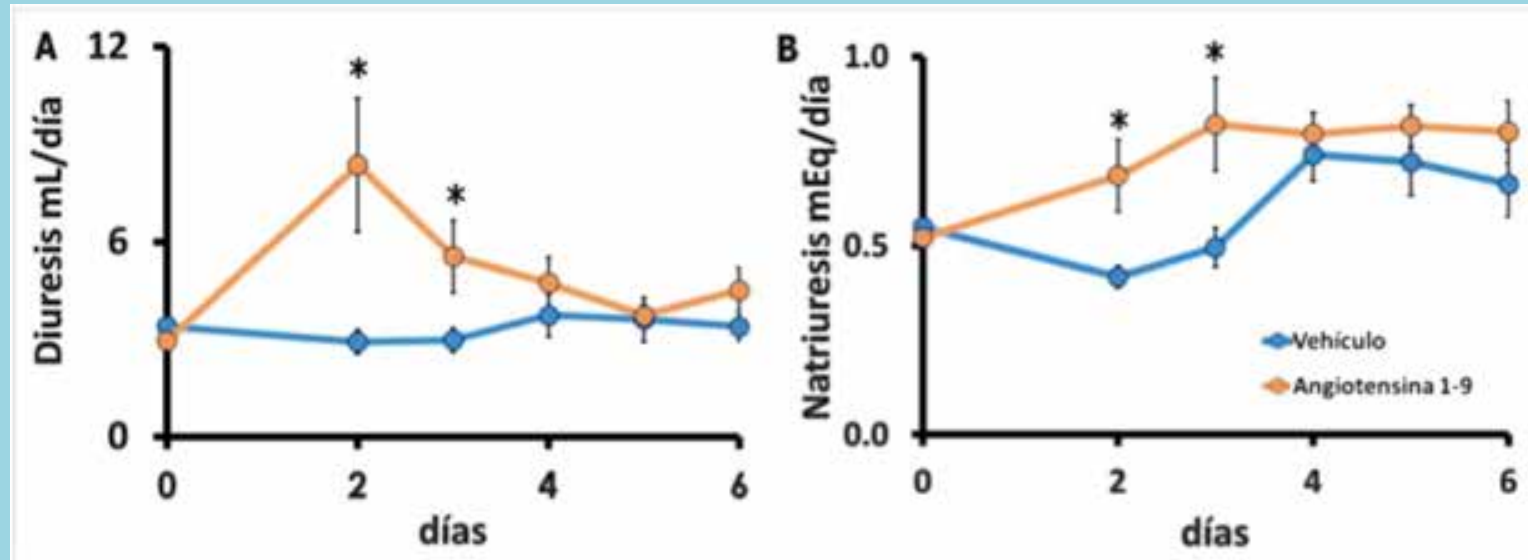

otra parte, Ang-(1-9) ejerció un efecto hipotensor al ser administrado en ratas normotensas. En nuestros distintos estudios se demuestra que la eficacia antihipertensiva de Ang-(1-9) se ejercería de manera directa y no estaría mediada por Ang-(1-7) producto de la transformación de Ang-(1-9) por acción de la ECA. Por lo tanto, el eje del SRA a través de la ECA2 y de Ang- (1-9) podría ser un importante blanco terapéutico de la HTA.

Recientemente, Flores-Muñoz et al, mostró en su modelo experimental de ratas "strole-prone" espontáneamente hipertensas (SPSHR) que Ang-(1-9) administrada en dosis de $100 \mathrm{ng} \cdot \mathrm{Kg}-1 \cdot \mathrm{min}-1$ no modificó la $\mathrm{PAS}^{22}$. Estos resultados difieren del efecto antihipertensivo observado para Ang-(1-9) probablemente, debido a la menor dosis de Ang-(1-9) usada en ratas SPSHR comparada con nuestros estudios de regresión de la HTA ${ }^{23}$.

Por otra parte, los resultados del presente estudio muestran que la administración crónica de Ang-(1-9), en un modelo experimental de ratas previamente hipertensas, logró revertir la hipertrofia cardíaca, destacando que este efecto tampoco fue mediado por Ang-(1-7). Éstos resultados complementan observaciones previas de nuestro grupo, que evidenciaron que Ang-(1-9) previno la $\mathrm{HTA}^{13} \mathrm{y}$ disminuyó la hipertrofia cardiaca tanto in vivo and in vitro ${ }^{10}$. Además, Flores et al (2011) apoyó el concepto que Ang(1-9) es un péptido con propiedades anti-hipertróficas, cuyo mecanismo de acción era vía el receptor tipo 2 de Angiotensina II (AT2R) ${ }^{24}$.

Junto con la regresión de la hipertrofia cardíaca ${ }^{10}$, Ang-(1-9) disminuyó la fibrosis cardíaca hipertensiva.
Ambos efectos estuvieron directamente relacionados a la administración de Ang-(1-9) y no mediados por Ang-(17). Éstos resultados son consistentes con estudios publicados que muestran que la admistración de Ang- (1-9) a ratas SPSHR disminuyó la fibrosis cardíaca ${ }^{22}$. El efecto antifibrótico fue bloqueado por el antagonista del AT2R. Además, Ang-(1-9) inhibió la proliferación de fibroblastos in vitro, cuyo efecto fue sensible al antagonista del AT2R, PD123319. Estos resultados muestran que la activación del AT2R por Ang-(1-9) tiene un importante efecto antifibrótico que puede implicar una acción directa sobre los fibroblastos 22 .

En relación al mecanismo de acción de Ang-(1-9), antecedentes previos señalan que ésta presenta propiedades vasoactivas al aumentar los niveles de eNOS y nitratos plasmáticos, sugiriendo que las acciones vasodilatadoSRA de Ang-(1-9) estarían mediadas por un incremento de Óxido Nítrico (NO, potente vasodilatador) ${ }^{25}$. Además, ha sido descrito que Ang-(1-9) potencia la liberación de bradicinina en células endoteliales aisladas de tejido cardíaco, lo que se relaciona con el aumento de la biodisponibilidad de $\mathrm{NO}$ en las células endoteliales ${ }^{22}$. Cuando las células endoteliales son tratadas con Ang-(1-9) y PD123,3 $3^{19}$ (Antagonista del receptor AT2) disminuye la biodisponibilidad de los niveles de NO en los animales de control ${ }^{22}$. Recientemente, en ratas espontáneamente hipertensas, se demostró que Ang-(1-9) disminuyó los niveles de colágeno cardiaco vía el receptor AT2 específicamente ${ }^{22}$. Estos antecedentes señalan que uno de los posibles mecanismos de acción de Ang-(1-9) podría ser 
Tabla 1. Efecto de Ang-(1-9) en la masa corporal y ventricular. Abreviaturas: $S=$ ratas normotensas, $S /$ Ang 1-9 = ratas normotensas tratadas con Ang-(1-9), Ang II = ratas hipertensas. Ang II/(1-9)= ratas hipertensas con infusión de Ang-(1-9), Ang II/(1-9)/A779 = ratas hipertensas con coadministración de Ang-(1-9) /A779. Se midió la masa corporal (MC) inicial y final, masa ventricular (MV), masa ventricular relativa (MVR), según el lago de la tibia (LT) para los distintos grupos experimentales. Los datos fueron expresados según Promedio \pm SEM. $N=7-10,{ }^{*} p \leq 0,05$ vs Sham, \# $p \leq 0,05$ vs Ang II, después de ANOVA para comparación entre grupos.

\begin{tabular}{|c|ccccc|}
\cline { 2 - 6 } \multicolumn{1}{c|}{} & S & S/Ang 1-9 & Ang II & Ang II/(1-9) & Ang II/(1-9)/A779 \\
\hline N & $\mathbf{1 0}$ & $\mathbf{8}$ & $\mathbf{9}$ & $\mathbf{9}$ & $\mathbf{7}$ \\
\hline MC inicial (g) & $205 \pm 2$ & $210 \pm 5$ & $206 \pm 3$ & $212 \pm 3$ & $208 \pm 1$ \\
MC final (g) & $318 \pm 8$ & $326 \pm 4$ & $305 \pm 12$ & $259 \pm 5 *$ & $267 \pm 17 *$ \\
MV (g) & $0.99 \pm 0.01$ & $1.00 \pm 0.02$ & $1.15 \pm 0.01 *$ & $0.99 \pm 0.02 *$ & $0.95 \pm 0.05 *$ \\
MVR (MV/LT, $\mathbf{m g} / \mathbf{c m})$ & $25.9 \pm 0.4$ & $26.4 \pm 0.9$ & $30.4 \pm 0.4 *$ & $26.5 \pm 0.4^{*}$ & $26.9 \pm 1.1 *$ \\
LT (cm) & $3.83 \pm 0.03$ & $3.78 \pm 0.02$ & $3.76 \pm 0.02$ & $3.75 \pm 0.05$ & $3.73 \pm 0.03$ \\
\hline
\end{tabular}

a través del AT2R y la liberación de NO. ${ }^{22}$ Se ha descrito la estimulación AT2R aumenta la síntesis de NO en las células endoteliales cardíacas, promoviendo la vasodilatación. Por otra parte, NO tiene efectos directos sobre el transporte tubular de sodio, inhibiendo su reabsorción ${ }^{26-27}$. Hay una fuerte evidencia de que los efectos del NO son mediados por el GMP cíclico extracelular del intersticio renal ${ }^{28-29}$. Respecto a nuestro estudio, Ang-(19) aumentó significativamente la natriuresis y diuresis en los animales normotensos. Éste es el primer informe que muestra los efectos de la Ang-(1-9) en la manipulación de sodio renal. Dado que la AT2R trabaja a través del NO mediando la vía de producir GMP cíclico ${ }^{30}$, es plausible que la Ang-(1-9) module la natriuresis a través de las acciones del NO en la vasculatura y/o túbulo renal.

Otros estudios demuestran que la activación de la AT2R en el riñón induce la respuesta natriurética ${ }^{31-32}$. Para llegar a esta conclusión, los estudios respectivos se basaron en modelos de ratas knock-out para el AT2R, que presentaron una respuesta anti-natriurética exagerada ${ }^{33}$. Adicionalmente, se ha observado que la activación de la AT2R promueve la natriuresis en ratas obesas Zucker (OZR), y la infusión de un agonista no peptídico AT2R (C21) aumenta el flujo de orina y la excreción urinaria de $\mathrm{Na}+{ }^{34}$.

Por otra parte, respecto al tono dopaminérgico renal que representa el 50-60\% de la natriuresis total, se ha demostrado que el AT2R interacciona con el receptor de tipo 1 en el túbulo proximal (D1R) ${ }^{35-36}$. Un aumento del AMP cíclico (cAMP) intracelular generado por la estimulación de D1R en el túbulo proximal, logra reclutar el AT2R a la membrana apical del túbulo proximal, generando una respuesta natriurética complementaria a $\mathrm{D} 1 \mathrm{R}^{37}$.

Por lo tanto, se sugiere que el efecto de la infusión aguda de Ang-(1-9) puede resultar en la activación AT2R en el túbulo proximal, que conduce a una mayor respuesta natriurética.

El hallazgo de que Ang-(1-9) estaría actuando en la regulación de sodio a nivel renal, abre nuevas áreas de investigación para el conocimiento de las acciones biológicas de Ang-(1-9) y aumentando sus efectos benéficos en el tratamiento de la HTA. Estos antecedentes establecen con mayor fuerza la idea de utilizar a Ang-(1-9) como una nueva estrategia terapeútica para el tratamiento de la HTA y sus efectos secundarios.

En conclusión, Ang-(1-9) redujo la hipertensión y el remodelamiento cardíaco en ratas hipertensas. En animales normotensos, se demostró que el tratamiento con Ang-(19) induce diuresis y natriuresis. Éste es el primer reporte que señala que el efecto de Ang-(1-9) está asociado a una regulación del sodio a nivel renal. 


\section{Referencias:}

1. MESSERLI F, WILLIAMS B, RITZ E. Essential hypertension, Lancet 2007, 370:591-603

2. TOUYZ R. Intracellular mechanisms involved in vascular remodelling of resistance arteries in hypertension: role of angiotensin II. Exp Physiol. 2005; 90: 449-455.

3. TIPNIS SR, HOOPER NM, HYDE R, KARRAN E, CHRISTIE G, TURNER AJ. A human homolog of angiotensin converting enzyme. Cloning and functional expression as a captopril-insensitive cardoxypeptidase. J. Biol. Chem 2000; 275: 33238-33243.

4. DONOGHUE M1, HSIEH F, BARONAS E, GODBOUT $\mathrm{K}$, GOSSELIN M, STAGLIANO N, et al. A novel angiotensin-converting enzyme related carboxypeptidase (ACE2) converts angiotensin I to angiotensin 1-9. Circ. 2000; 87: e1-e9.

5. VICKERS C1, HALES P, KAUSHIK V, DICK L, GAVIN J, TANG J, et al. Hydrolisis of biological peptides by human angiotensin-converting enzyme-related carboxypeptidase (ACE2). J. Biol. Chem 2002; 277: 14838-14843.

6. RICE GI1, THOMAS DA, GRANT PJ, TURNER AJ, HOOPER NM. Evaluation of angiotensin-converting enzyme (ACE), its homologue ACE2 and neprilysin in angiotensin peptide metabolism. Biochem $\mathrm{J}$ 2004; 383:45-51.

7. SANTOS RA1, FERREIRA AJ, SIMÕES E SILVA AC. Recent advances in the angiotensin- converting enzyme2-angiotensin-(1-7)-Mas axis. Exp Physiol 2008; 93: 519-527.

8. FERRARIO C. ACE2: more of Ang-(1-7) or less Ang II?. Curr Opin Nephrol Hypertens 2011; 20:1-6.

9. OCARANZA MP, GODOY I, JALIL JE, VARAS M, COLLANTES P, PINTO M, et al. Enalapril attenuates downregulation of angiotensin-converrung enzyme 2 in the late phase of ventricular dysfunction in myocardial infracted rat. Hypertension. 2006; 48:572-578.

10. OCARANZA MP1, LAVANDERO S, JALIL JE, MOYA J, PINTO M, NOVOA U, et al. Angiotensin-(1-9) regulates cardiac hypertrophy in vivo and in Vitro. Journal of Hypertension 2010; 28:1054-1064.
11. OCARANZA MP1, RIVERA P, NOVOA U, PINTO M, GONZÁLEZ L, CHIONG M, et al. Rho kinase inhibition activates the homologous angiotensin-converting enzyme-angiotensin-(1-9) axis in experimental hypertension. J Hypertension 2011; 29:706-715.

12. OCARANZA M, MOYA J, PINTO M, ESCUDEROS N, VALENZUELA F, et al. Menores niveles tisulares de la enzima convertidora de angiotensina I homologa (ECA-2) y angiotensina-(1-9) están asociados a mayor remodelamiento de la pared aórtica de las ratas hipertensas. Rev Chil Cardiol 2010; 29:69-82.

13. LAGOS T, ESCUDERO N, NOVOA U, GODOY I, CHIONG $\mathrm{M}$, et al. Angitensina 1-9 previene la hipertensión arterial y el remodelamiento de la pared aortica inducido por Angiotensina II. Rev Chil Cardiol 2009; 38:333-334.

14. MOYA J, MORALES C, GODOY I, NOVOA U, et al. Angiotensina-(1-9) ejerce un efecto antihipertensivo, regresa el remodelamiento cardiaco y mejora la fracción de eyección en la hipertensión arterial experimental. Rev Chil Cardiol 2011;30: 83-84.

15. PIDDO A, SÁNCHEZ I, SAPÁG-HAGAR M, CORBALÁN $\mathrm{R}$, et al. Cyclic AMP- dependent protein kinase and mechanical heart function in ventricular hypertrophy induced by pressure overload or secondary to myocardial infarction. J Mol Cell Cardiol 1996; 28:1073-1083.

16. IGASE M, STRAWN W, GALLAGHER P, GEARY R, FERRARIO C. Angiotensin II AT1 receptors regulate ACE2 and angiotensin-(1-7) expression in the aorta of spontaneously hypertensive rats. Am J Physiol Heart Circ Physiol 2005; 289:H1013-H1019.

17. CUSHMAN DW, CHEUNG HS. Concentrations of angiotensin-converting enzyme in tissues of the rat. Biochim Biophys Acta 1971; 250: 261-265.

18. NAKAMURA K, FUSHIMI K, KOUCHI H, MIHARA K, MIYAZAKI M. Inhibitory effects of antioxidants on neonatal rat cardiac myocyte hypertrophy induced by tumor necrosis factor- and angiotensin II. Circulation 1998; 98: 794799. 
19. DUSSAILlANT G, JALIL JE, CESPEDES C. Amiodarone protection against myocardial injury and fibrosis induced by isoprenaline is abolished by thyroid hormone. Cardiovascular. 1994; 28: 1008-1013.

20. JALIL JE, JANICKI JS, PICK R, WEBER KT. Coronary vascular remodeling and myocardial fibrosis in the rat with renovascular hypertension. Response to captopril. Am J Hypertens. 1991; 4: 51-55.

21. MOYA J, NOVOA U, GODOY I, CHIONG M, LAVANDEROS, et al. Angiotensina-(1-9) es efectiva como agente antihipertensivo que disminuye el remodelamiento cardiovascular independiente de los niveles de eca,Angiotensina II y del receptor de Angiotensina 1-7. Libro de Resúmenes, XLVII Congreso Chileno de Cardiología y Cirugía Cardiovascular. 2010; 29: 77.

22. FLORES-MUNOZ M1, WORK LM, DOUGLAS K, DENBY L, DOMINICZAK AF, GRAHAM D, et al Angiotensin-(1-9) Attenuates Cardiac Fibrosis in the Stroke-Prone Spontaneously Hypertensive Rat via the Angiotensin Type 2 Receptor Hypertension; 2012; 59: 300-307.

23. OCARANZA M.P, MOYA J, BARRIENTOS V, ALZAMORA R, HEVIA D, MORALES C, et al. (2014) Angiotensin-(1-9) Reverses Experimental Hypertension and Cardiovascular Damage by Inhibition of the Angiotensin Converting Enzyme/ Ang Ii Axis. J Hypertens. 2014; 32: 771-783.

24. FLORES-MUÑOZ M, SMITH N, HAGGERTY C, MILLIGAN G, NICKLIN S. Angiotensin1-9 antagonises pro-hypertrophic signalling in cardiomyocytes via the angiotensin type 2 receptor. J Physiol; 2011, 589.4: 939-951.

25. MOYA J, HEVIA D, GARCIA L, NOVOA U, et al. El efecto anti-hipertensivo de Angiotensina-(1-9) esta mediado por mayor expresión de eNOS en la pared aórtica de ratas hipertensas. Rev Chil Cardiol, Libro resúmenes 2011; 84.

26. EVANS RG, MAJID DS, EPPEL GA. Mechanisms mediating pressure natriuresis: what we know and what we need to find out. Clin Exp Pharmacol Physiol. 2005; 32: 400-409.

27. ORTIZ PA, GARVIN JL. Role of nitric oxide in the regulation of nephron transport. Am J Physiol Renal Physiol. 2002; 282: F777-784.
28. AHMED F, KEMP BA, HOWELL NL, SIRAGY HM, CAREY RM. Extracellular renal guanosine cyclic 3'5'-monophosphate modulates nitric oxide and pressureinduced natriuresis. Hypertension. 2007; 50: 958-963.

29. LIEB DC, KEMP BA, HOWELL NL, GILDEA JJ, CAREY RM. Reinforcing feedback loop of renal cyclic guanosine 3' 5 ' -monophosphate and interstitial hydrostatic pressure in pressure-natriuresis. Hypertension. 2009; 54: 1278-1283

30. CAREY RM, JIN X, WANG Z, SIRAGY HM. Nitric oxide: a physiological mediator of the type 2 (AT2) angiotensin receptor. Acta Physiol Scand. 2000; 168: 65-71.

31. HAKAM AC, HUSSAIN T. Renal angiotensin II type-2 receptors are upregulated and mediate the candesartan-induced natriuresis/diuresis in obese Zucker rats. Hypertension. 2005; 45: 270-275.

32. HAKAM AC, HUSSAIN T. Angiotensin II AT2 receptors inhibit proximal tubular $\mathrm{Na}+\mathrm{K}+-\mathrm{ATPase}$ activity via a $\mathrm{NO} /$ cGMP-dependent pathway. Am J Physiol Renal Physiol. 2006; 290: F1430-F1436.

33. SIRAGY HM, INAGAMI T, ICHIKI T, CAREY RM. Sustained hypersensitivity to angiotensin II and its mechanism in mice lacking the subtype-2 (AT2) angiotensin receptor. Proc Natl Acad Sci U S A. 1999; 96:6506-6510.

34. ALI Q, HUSSAIN T. AT(2) receptor non-peptide agonist C21 promotes natriuresis in obese Zucker rats. Hypertens Res. 2012;35:654-660

35. GILDEA JJ, WANG X, SHAH N, TRAN H, SPINOSA M, VAN SCIVER R, et al. Dopamine and angiotensin type 2 receptors cooperatively inhibit sodium transport in human renal proximal tubule cells. Hypertension. 2012; 60: 396-403.

36. SALOMONE LJ, HOWELL NL, MCGRATH HE, KEMP BA, KELLER SR, GILDEA JJ, et al. Intrarenal dopamine D1-like receptor stimulation induces natriuresis via an angiotensin type-2 receptor mechanism. Hypertension. 2007; 49: 155-161.

37. PADIA SH, KEMP BA, HOWELL NL, KELLER SR, GILDEA JJ, CAREY RM. Mechanisms of Dopamine D1 and Angiotensin AT2 receptor interaction in natriuresis. Hypertension. 2012; 59: 437-445. 